EPJ Web of Conferences 80, 00053 (2014)

DOI: $10.1051 /$ epj conf/20148000053

(C) Owned by the authors, published by EDP Sciences, 2014

\title{
Electroweak chiral Lagrangians and the Higgs properties at the one-loop level
}

\author{
J.J. Sanz-Cillero ${ }^{1, a}$ \\ ${ }^{1}$ Departamento de Física Teórica and Instituto de Física Teórica, IFT-UAM/CSIC \\ Universidad Autónoma de Madrid, C/ Nicolás Cabrera 13-15, \\ Cantoblanco, 28049 Madrid, Spain
}

\begin{abstract}
In these proceedings we explore the use of (non-linear) electroweak chiral Lagrangians for the description of possible beyond the Standard Model (BSM) strong dynamics in the electroweak (EW) sector. Experimentally one observes an approximate EW symmetry breaking pattern $S U(2)_{L} \times S U(2)_{R} / S U(2)_{L+R}$. Quantum Chromodynamics (QCD) shows a similar chiral structure [1] and, in spite of the differences (in the EW theory $S U(2)_{L} \times U(1)_{Y}$ is gauged), it has served for years as a guide for this type of studies [2-4]. Examples of one-loop computations in the low-energy effective theory and the theory including the first vector $(\mathrm{V})$ and axial-vector $(\mathrm{A})$ resonances are provided, yielding, respectively, predictions for $\gamma \gamma \rightarrow Z_{L} Z_{L}, W_{L}^{+} W_{L}^{-}$and the oblique parameters $S$ and $T$.
\end{abstract}

\section{Introduction: strong dynamics and chiral Lagrangians}

A non-linear realization of the EW would-be Goldstone bosons (WBGBs) is considered to build the EW low-energy effective field theory (EFT), which is described by an EW chiral Lagrangian with a light Higgs (ECLh). It includes the Standard Model (SM) content: the EW Goldstones $w^{a}$, the EW gauge bosons $W_{\mu}^{a}$ and $B_{\mu}$ and a singlet Higgs $h$ (the fermion sector is not discussed here). In particular, in Sec. 2 we explain the chiral counting in the ECLh $[5,6]$ and provide an example of a next-to-leading order (NLO) computation: we calculate $\gamma \gamma \rightarrow W_{L}^{+} W_{L}^{-}, Z_{L} Z_{L}$ within this framework up to the one-loop level [5] at energies below new possible composite resonances, $\sqrt{s} \ll \Lambda_{\mathrm{ECLh}} \sim \min \left\{M_{R}, 4 \pi v\right\}$ (with $v=\left(\sqrt{2} G_{F}\right)^{-1 / 2}$ and $\left.4 \pi v \simeq 3 \mathrm{TeV}\right)$. Analogous works on $W W$-scattering can be found in Refs. [7].

However, in the case of having heavy composite resonance, the EFT stops being valid when the energy becomes of the order of their masses (expected to be of the order of $M_{R} \sim 4 \pi v \sim 3 \mathrm{TeV}$ ). One has to introduce these new degrees of freedom in our EW Lagrangian following a procedure analogous to that in QCD [8]. Likewise, under reasonable ultraviolet (UV) completion hypotheses like, e.g., the Weinberg sum-rules (WSRs) fulfilled by certain types of theories [9-13], one can make predictions on low-energy observables. In Sec. 3 we write down the relevant $S U(2)_{L} \times S U(2)_{R}$ invariant Lagrangian including the SM content and a multiplet of V and A resonances and extract one-loop limits on the resonance masses and the Higgs coupling $g_{h W W}$ [14] from the experimental values of oblique parameters $S$ and $T$ [15]. Alternative one-loop analyses can be found in Refs. [10, 16].

\footnotetext{
ae-mail: juanj.sanz@uam.es

I would like to thank the organizers for their work and the lively discussion during the workshop; also for their patience. This work is partially supported by the Spanish Government and ERDF funds from the European Commission [FPA2010-17747, FPA2013-44773-P, SEV-2012-0249, CSD2007-00042] and the Comunidad de Madrid [HEPHACOS S2009/ESP-1473].
} 


\section{Low-energy EFT: ECLh and one-loop $\gamma \gamma \rightarrow W_{L}^{a} W_{L}^{b}$ scattering}

The Higgs boson does not enter in the SM at tree-level in these processes (where one also has $\left.\mathcal{M}(\gamma \gamma \rightarrow Z Z)_{\mathrm{SM}}^{\text {tree }}=0\right)$. Nevertheless, one can search for new physics by studying the one-loop corrections [5], which are sensitive to deviations from the SM in the Higgs boson couplings. Our analysis [5] is performed in the Landau gauge and making use of the Equivalence Theorem (Eq.Th.) [17],

$$
\mathcal{M}\left(\gamma \gamma \rightarrow W_{L}^{a} W_{L}^{b}\right) \simeq-\mathcal{M}\left(\gamma \gamma \rightarrow w^{a} w^{b}\right),
$$

valid in the energy regime $m_{W}^{2}, m_{Z}^{2} \ll s$. The EW gauge boson masses $m_{W, Z}$ are then neglected in our computation. Furthermore, since $m_{h} \sim m_{W, Z} \ll 4 \pi v \simeq 3 \mathrm{TeV}$ we also neglect $m_{h}$ in our calculation. In summary, the applicability range in [5] is

$$
m_{W}^{2}, m_{Z}^{2}, m_{h}^{2} \stackrel{\text { Eq.Th. }}{\ll} \quad s, t, u \stackrel{\text { EFT }}{\ll} \Lambda_{\mathrm{ECLh}}^{2},
$$

with the upper limit given by the EFT cut-off $\Lambda_{\mathrm{ECLh}}$, expected to be of the order of $4 \pi v \simeq 3 \mathrm{TeV}$ or the mass of possible heavy BSM particles.

The WBGBs are described by a matrix field $U$ that takes values in the $S U(2)_{L} \times S U(2)_{R} / S U(2)_{L+R}$ coset, and transforms as $U \rightarrow L U R^{\dagger}[2,3]$. The relevant ECLh with the basic building blocks is

$$
\begin{aligned}
& U=u^{2}=1+i w^{a} \tau^{a} / v+O\left(w^{2}\right), D_{\mu} U=\partial_{\mu} U+i \hat{W}_{\mu} U-i U \hat{B}_{\mu}, V_{\mu}=\left(D_{\mu} U\right) U^{\dagger}, u^{\mu}=-i u^{\dagger} D^{\mu} U u^{\dagger}, \\
& \hat{W}_{\mu \nu}=\partial_{\mu} \hat{W}_{v}-\partial_{\nu} \hat{W}_{\mu}+i\left[\hat{W}_{\mu}, \hat{W}_{v}\right], \quad \hat{B}_{\mu v}=\partial_{\mu} \hat{B}_{v}-\partial_{v} \hat{B}_{\mu}, \quad \hat{W}_{\mu}=g W_{\mu}^{a} \tau^{a} / 2, \hat{B}_{\mu}=g^{\prime} B_{\mu} \tau^{3} / 2,
\end{aligned}
$$

with well-defined trasnformation properties $[3,5,14]$. Two particular parametrizations of the unitary matrix $U$ (exponential and spherical) were considered in [5], both leading to the same predictions for the physical (on-shell) observables. ${ }^{1}$ We consider the counting $\partial_{\mu}, m_{W}, m_{Z}, m_{h} \sim O(p), D_{\mu} U, V_{\mu} \sim$ $O(p)$ and $\hat{W}_{\mu \nu}, \hat{B}_{\mu \nu} \sim O\left(p^{2}\right)[5,6]$. We require the ECLh Lagrangian to be CP invariant, Lorentz invariant and $S U(2)_{L} \times U(1)_{Y}$ gauge invariant. Here we focus ourselves on the relevant terms for $\gamma \gamma \rightarrow w^{a} w^{b}$ at leading order (LO) $-O\left(p^{2}\right)$ - and NLO in the chiral counting $-O\left(p^{4}\right)-[3,5]$ :

$$
\begin{aligned}
& \mathcal{L}_{2}=-\frac{1}{2 g^{2}}\left\langle\hat{W}_{\mu \nu} \hat{W}^{\mu v}\right\rangle-\frac{1}{2 g^{\prime 2}}\left\langle\hat{B}_{\mu \nu} \hat{B}^{\mu \nu}\right\rangle+\frac{v^{2}}{4}\left[1+2 a \frac{h}{v}+b \frac{h^{2}}{v^{2}}\right]\left\langle D^{\mu} U^{\dagger} D_{\mu} U\right\rangle+\frac{1}{2} \partial^{\mu} h \partial_{\mu} h+\ldots, \\
& \mathcal{L}_{4}=a_{1} \operatorname{Tr}\left(U \hat{B}_{\mu \nu} U^{\dagger} \hat{W}^{\mu v}\right)+i a_{2} \operatorname{Tr}\left(U \hat{B}_{\mu \nu} U^{\dagger}\left[V^{\mu}, V^{\nu}\right]\right)-i a_{3} \operatorname{Tr}\left(\hat{W}_{\mu \nu}\left[V^{\mu}, V^{\nu}\right]\right)-\frac{c_{\gamma}}{2} \frac{h}{v} e^{2} A_{\mu v} A^{\mu \nu}+\ldots
\end{aligned}
$$

where $\langle X\rangle$ stands for the trace of the $2 \times 2$ matrix $X$, one has the photon field strength $A_{\mu \nu}=\partial_{\mu} A_{\nu}-\partial_{v} A_{\mu}$ and the dots stand for operators not relevant within our approximations for $\gamma \gamma$-scattering [5].

The amplitudes $\mathcal{M}\left(\gamma\left(k_{1}, \epsilon_{1}\right) \gamma\left(k_{2}, \epsilon_{2}\right) \rightarrow w^{a}\left(p_{1}\right) w^{b}\left(p_{2}\right)\right)$, with $w^{a} w^{b}=z z, w^{+} w^{-}$, have the structure

$$
\mathcal{M}=i e^{2}\left(\epsilon_{1}^{\mu} \epsilon_{2}^{\nu} T_{\mu \nu}^{(1)}\right) A(s, t, u)+i e^{2}\left(\epsilon_{1}^{\mu} \epsilon_{2}^{\nu} T_{\mu \nu}^{(2)}\right) B(s, t, u),
$$

written in terms of the two independent Lorentz structures $T_{\mu \nu}^{(1,2)} \sim O\left(p^{2}\right)$ involving the external momenta, which can be found in [5]. The Mandelstam variables are defined as $s=\left(p_{1}+p_{2}\right)^{2}$, $t=\left(k_{1}-p_{1}\right)^{2}$ and $u=\left(k_{1}-p_{2}\right)^{2}$ and the $\epsilon_{i}$ 's are the polarization vectors of the external photons.

In dimensional regularization, our NLO computation of the $\mathcal{M}\left(\gamma \gamma \rightarrow w^{a} w^{b}\right)$ amplitudes can be systematically sorted out in the form [5]

$$
\mathcal{M}=\mathcal{M}_{\mathrm{LO}}+\mathcal{M}_{\mathrm{NLO}} \sim \underbrace{O\left(e^{2}\right)}_{\text {LO, tree }}+(\underbrace{O\left(e^{2} \frac{p^{2}}{16 \pi^{2} v^{2}}\right)}_{\mathrm{NLO}, 1-\text { loop }}+\underbrace{O\left(e^{2} \frac{a_{i} p^{2}}{v^{2}}\right)}_{\mathrm{NLO}, \text { tree }}),
$$

\footnotetext{
${ }^{1}$ Other representations have been recently studied in Ref. [18].
} 
Table 1. Running $\frac{d a_{i}^{r}}{d \ln \mu}=-\frac{\Gamma_{a_{i}}}{16 \pi^{2}}$ of the relevant ECLh parameters and their combinations appearing in the six selected observables. The third column provides the corresponding running for the Higgsless EW chiral Lagrangian (ECL) case [4]. For the sake of completeness, we have added the running of the ECLh parameters $a_{4}^{r}$ and $a_{5}^{r}$, which has been recently determined in the one-loop analysis of $W W$-scattering within the framework of chiral Lagrangians [7]. One can see that in the SM limit $(a=b=1)$ these $\mathcal{L}_{4}$ coefficients do not run, in agreement with the fact that these higher order operators are absent in the SM.

\begin{tabular}{c|c|c} 
& ECLh & ECL (Higgsless) \\
\hline$\Gamma_{a_{1}-a_{2}+a_{3}}$ & 0 & 0 \\
\hline$\Gamma_{c_{\gamma}}$ & 0 & - \\
\hline$\Gamma_{a_{1}}$ & $-\frac{1}{6}\left(1-a^{2}\right)$ & $-\frac{1}{6}$ \\
\hline$\Gamma_{a_{2}-a_{3}}$ & $-\frac{1}{6}\left(1-a^{2}\right)$ & $-\frac{1}{6}$ \\
\hline$\Gamma_{a_{4}}$ & $\frac{1}{6}\left(1-a^{2}\right)^{2}$ & $\frac{1}{6}$ \\
\hline$\Gamma_{a_{5}}$ & $\frac{1}{8}\left(b-a^{2}\right)^{2}+\frac{1}{12}\left(1-a^{2}\right)^{2}$ & $\frac{1}{12}$ \\
\hline
\end{tabular}

where $e \sim O(p / v)$ and $A$ and $B$ are given up to NLO by [5]

$$
\begin{array}{rlrl}
A(\gamma \gamma \rightarrow z z)_{\mathrm{LO}} & =B(\gamma \gamma \rightarrow z z)_{\mathrm{LO}}=0, & \\
A(\gamma \gamma \rightarrow z z)_{\mathrm{NLO}} & =\frac{2 a c_{\gamma}^{r}}{v^{2}}+\frac{\left(a^{2}-1\right)}{4 \pi^{2} v^{2}}, & B(\gamma \gamma \rightarrow z z)_{\mathrm{NLO}}=0, \\
A\left(\gamma \gamma \rightarrow w^{+} w^{-}\right)_{\mathrm{LO}} & =2 s B\left(\gamma \gamma \rightarrow w^{+} w^{-}\right)_{\mathrm{LO}}=-\frac{1}{t}-\frac{1}{u}, & \\
A\left(\gamma \gamma \rightarrow w^{+} w^{-}\right)_{\mathrm{NLO}} & =\frac{2 a c_{\gamma}^{r}}{v^{2}}+\frac{\left(a^{2}-1\right)}{8 \pi^{2} v^{2}}+\frac{8\left(a_{1}^{r}-a_{2}^{r}+a_{3}^{r}\right)}{v^{2}}, \quad B\left(\gamma \gamma \rightarrow w^{+} w^{-}\right)_{\mathrm{NLO}}=0 .
\end{array}
$$

The term with $c_{\gamma}^{r}$ comes from the Higgs tree-level exchange in the $s$-channel, the term proportional to $\left(a^{2}-1\right)$ comes from the one-loop diagrams with $\mathcal{L}_{2}$ vertices, and the Higgsless operators in Eq. (5) yield the tree-level contribution to $\gamma \gamma \rightarrow w^{+} w^{-}$proportional to $\left(a_{1}-a_{2}+a_{3}\right)$. Independent diagrams are in general UV divergent. However, in dimensional regularization, the final one-loop amplitude turns out to be UV finite and one has $a_{1}^{r}-a_{2}^{r}+a_{3}^{r}=a_{1}-a_{2}+a_{3}, c_{\gamma}^{r}=c_{\gamma}$ [5], as in the Higgsless case [19].

In order to pin down each of the relevant combinations of ECLh couplings in Eq. (7) ( $a, c_{\gamma}^{r}$ and $a_{1}^{r}-a_{2}^{r}+a_{3}^{r}$ ) one must combine our $\gamma \gamma$-scattering analysis with other observables that depend on this same set of parameters. It is not difficult to find that other processes involving photons depend on these parameters. In Ref. [5] we computed 4 more observables of this kind: the $h \rightarrow \gamma \gamma$ decay width (depending on $a$ and $c_{\gamma}$ ), the oblique $S$-parameter (depending on $a$ and $a_{1}$ ), and the $\gamma^{*} \rightarrow w^{+} w^{-}$ (depending on $a$ and $a_{2}-a_{3}$ ) and $\gamma^{*} \gamma \rightarrow h$ (depending on $c_{\gamma}$ ) electromagnetic form-factors. The one-loop contribution in these six relevant amplitudes is found to be UV-divergent in some cases. These divergences are absorbed by means of the generic $O\left(p^{4}\right)$ renormalizations $a_{i}^{r}(\mu)=a_{i}+\delta a_{i}$. As expected, the renormalization in the six observables gives a fully consistent set of renormalization conditions and fixes the running of the renormalized couplings in the way given in Table 1. 

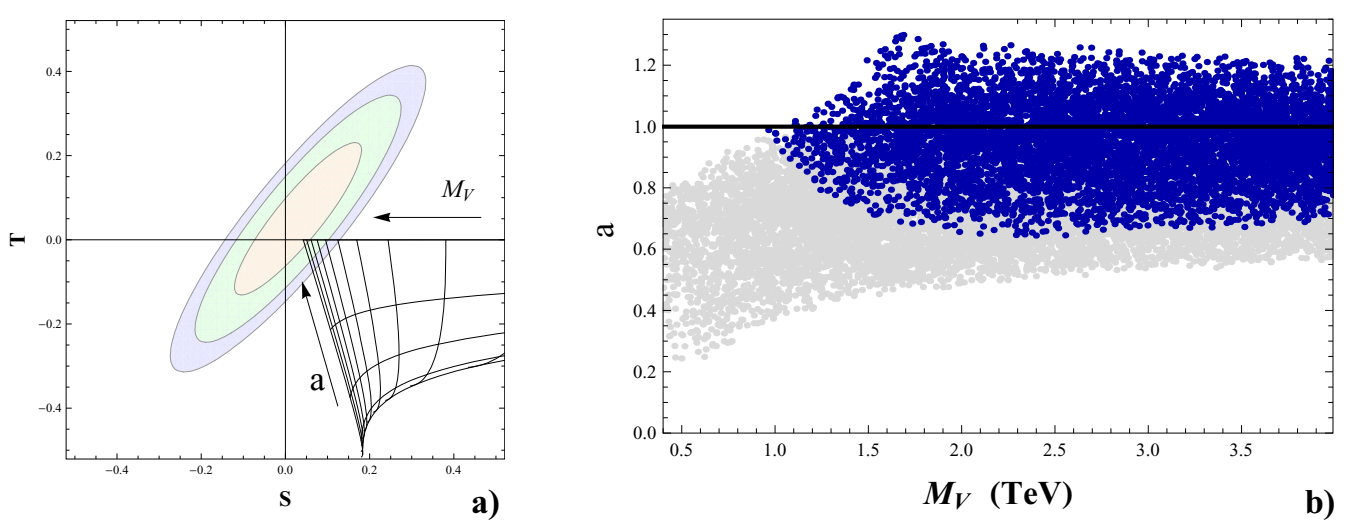

Figure 1. a) NLO determinations of $S$ and $T$, imposing the two WSRs. The approximately vertical curves correspond to constant values of $M_{V}$, from 1.5 to $6.0 \mathrm{TeV}$ at intervals of $0.5 \mathrm{TeV}$. The approximately horizontal curves have constant values of $a$ : $0.00,0.25,0.50,0.75,1.00$. The arrows indicate the directions of growing $M_{V}$ and $a$. The ellipses give the experimentally allowed regions at $68 \%$ (orange), $95 \%$ (green) and $99 \%$ (blue) confidence level (CL). b) Scatter plot for the $68 \%$ CL region, in the case when only the first WSR is assumed. The dark blue and light gray regions correspond, respectively, to $0.2<M_{V} / M_{A}<1$ and $0.02<M_{V} / M_{A}<0.2$.

\section{Impact of spin-1 composite resonances on the oblique parameters}

One can extend the range of validity and predictability of the ECLh by adding possible new states to the theory. Thus, the lightest V and A resonances are added to the EW Lagrangian in Ref. [14] in order to describe the oblique parameters $S$ and $T$ [9]. The relevant EW chiral invariant Lagrangian is given by the kinetic and Yang-Mills terms and the interactions [14] ${ }^{2,3}$

$$
\begin{aligned}
\mathcal{L}= & \frac{v^{2}}{4}\left\langle u_{\mu} u^{\mu}\right\rangle\left(1+\frac{2 a}{v} h\right)+\frac{F_{V}}{2 \sqrt{2}}\left\langle V_{\mu v} f_{+}^{\mu v}\right\rangle+\frac{i G_{V}}{2 \sqrt{2}}\left\langle V_{\mu \nu}\left[u^{\mu}, u^{v}\right]\right\rangle \\
& +\frac{F_{A}}{2 \sqrt{2}}\left\langle A_{\mu v} f_{-}^{\mu v}\right\rangle+\sqrt{2} \lambda_{1}^{h A} \partial_{\mu} h\left\langle A^{\mu v} u_{v}\right\rangle .
\end{aligned}
$$

In order to compute $S$ and $T$ up to the one-loop level we use the dispersive representations [9, 14],

$$
S=\frac{16 \pi}{g^{2} \tan \theta_{W}} \int_{0}^{\infty} \frac{\mathrm{dt}}{t}\left[\rho_{S}(t)-\rho_{S}(t)^{\mathrm{SM}}\right], \quad T=\frac{4 \pi}{g^{\prime 2} \cos ^{2} \theta_{W}} \int_{0}^{\infty} \frac{\mathrm{dt}}{t^{2}}\left[\rho_{T}(t)-\rho_{T}(t)^{\mathrm{SM}}\right],
$$

with $\rho_{S}(t)$ the spectral function of the $W^{3} B$ correlator $[9,21]$ and $\rho_{T}(t)$ the spectral function of the difference of the neutral and charged Goldstone self-energies [14]. The calculation of $T$ above has been simplified by means of the Ward-Takahashi relation $T=Z^{\left(w^{+}\right)} / Z^{\left(w^{0}\right)}-1$ [20]. Only the lightest two-particle cuts have been considered in $\rho_{S}(t)$ and $\rho_{T}(t)$, respectively, $\{w w, w h\}$ and $\{B w, B h\}$. Since $\rho_{S}(t) \stackrel{\mathrm{SM}}{\longrightarrow \rightarrow \infty} \longrightarrow 0$, the convergence of the Peskin-Takeuchi sum-rule requires $\rho_{S}(t) \stackrel{t \rightarrow \infty}{\longrightarrow} 0$. Furthermore, assuming that weak isospin and parity are good symmetries of the BSM strong dynamics, the $W^{3} B$ correlator is proportional to the difference of the vector and axial-vector two-point Green's functions [9]. In asymptotically-free gauge theories this difference vanishes at $s \rightarrow \infty$ as $1 / s^{3}$ [12], implying the (tree-level) LO WSRs [13],

$$
F_{V}^{2}-F_{A}^{2}=v^{2} \quad(1 \mathrm{st} \mathrm{WSR}), \quad F_{V}^{2} M_{V}^{2}-F_{A}^{2} M_{A}^{2}=0 \quad \text { (2nd WSR). }
$$

\footnotetext{
${ }^{2}$ Here we follow the notation $f_{ \pm}^{\mu v}=u^{\dagger} \hat{W}^{\mu v} u \pm u \hat{B}^{\mu v} u^{\dagger}$ from Ref. [14, 21], where there is a global sign difference with [5] in the definitions of $\hat{W}_{\mu}$ and $\hat{B}_{\mu}$. The spin-1 resonances are described in the antisymmetric tensor formalism [8].

${ }^{3}$ In other works, the coupling $a$ can be found with the notation $\kappa_{W}$ and $\omega$ [14] or $\kappa_{V}$ [22].
} 
Table 2. Allowed range for the $M_{V}$ and $a$ at the $68 \%$ CL for the two-WSRs (where $V$ and $A$ are very degenerate since $M_{V}^{2} / M_{A}^{2}=a$ in this case) and only-1st-WSR cases (for various values $M_{V} / M_{A}$ ). In the last line we also impose the restriction ${ }^{\dagger} M_{V}>1 \mathrm{TeV}$.

\begin{tabular}{c|c|c} 
& $a$ & $M_{V}$ \\
\hline two WSRs & $0.97-1$ & $>5 \mathrm{TeV}$ \\
\hline Only 1st WSR: $\quad 0.2<M_{V} / M_{A}<1$ & $0.6-1.3$ & $>1 \mathrm{TeV}$ \\
$0.5<M_{V} / M_{A}<1$ & $0.84-1.30$ & $>1.5 \mathrm{TeV}$ \\
$M_{V} / M_{A}=1$ & $0.97-1.30$ & $>1.8 \mathrm{TeV}$ \\
$\left(M_{V}>1 \mathrm{TeV}\right)^{\dagger} \quad 1<M_{V} / M_{A}<2$ & $0.7-1.9$ & $>1 \mathrm{TeV}^{\dagger}$ \\
\hline
\end{tabular}

However, although the 1st WSR is expected to be true in gauge theories with non-trivial ultraviolet fixed points $[10,11]$, the 2 nd WSR is questionable in some of these models. Thus, two alternative scenarios are studied in Ref. [14]: one assuming the two WSRs and another assuming just the 1st WSR. At tree-level one has the the LO determinations [9, 14, 21]

$$
\begin{array}{ll}
S_{\mathrm{LO}}=4 \pi\left(\frac{F_{V}^{2}}{M_{V}^{2}}-\frac{F_{A}^{2}}{M_{A}^{2}}\right)=\frac{4 \pi v^{2}}{M_{V}^{2}}\left(1+\frac{M_{V}^{2}}{M_{A}^{2}}\right) \quad(1 \mathrm{st} \& 2 \text { nd WSR }) \\
S_{\mathrm{LO}}=4 \pi\left\{\frac{v^{2}}{M_{V}^{2}}+F_{A}^{2}\left(\frac{1}{M_{V}^{2}}-\frac{1}{M_{A}^{2}}\right)\right\}>\frac{4 \pi v^{2}}{M_{V}^{2}} \quad\left(1 \mathrm{st} \text { WSR \& } M_{V}<M_{A}\right) .
\end{array}
$$

In the first case, the two WSRs imply $M_{V}<M_{A}$ and determine $F_{V}$ and $F_{A}$ in terms of the resonance masses $[8,9,14,21]$. In the second case, it is not possible to extract a definite prediction with just the 1st WSR but one can still derive the inequality above if one assumes a similar mass hierarchy $M_{V}<M_{A}$. On the other hand, this inequality flips direction if $M_{A}<M_{V}$ or turns into an equality in the degenerate case $M_{V}=M_{A}$ [14]. At NLO the computed $W^{3} B$ correlator is given by the $w w$ and $h w$ cuts, whose contributions to the $\rho_{S}(t)$ spectral function would have an unphysical grow at high energies unless $F_{V} G_{V}=v^{2}$ and $F_{A} \lambda_{1}^{h A}=a v[8,14,21]$. Thus, we obtain the NLO prediction [14]

$$
\begin{aligned}
S= & 4 \pi v^{2}\left(\frac{1}{M_{V}^{2}}+\frac{1}{M_{A}^{2}}\right) \\
& +\frac{1}{12 \pi}\left[\log \frac{M_{V}^{2}}{m_{H}^{2}}-\frac{11}{6}+\frac{M_{V}^{2}}{M_{A}^{2}} \log \frac{M_{A}^{2}}{M_{V}^{2}}-\frac{M_{V}^{4}}{M_{A}^{4}}\left(\log \frac{M_{A}^{2}}{m_{S_{1}}^{2}}-\frac{11}{6}\right)\right] \quad \text { (1st \& 2nd WSR), } \\
S> & \frac{4 \pi v^{2}}{M_{V}^{2}}+\frac{1}{12 \pi}\left[\log \frac{M_{V}^{2}}{m_{H}^{2}}-\frac{11}{6}-a^{2}\left(\log \frac{M_{A}^{2}}{m_{S_{1}}^{2}}-\frac{17}{6}+\frac{M_{A}^{2}}{M_{V}^{2}}\right)\right], \quad\left(1 \mathrm{st} \text { WSR \& } M_{V}<M_{A}\right) .
\end{aligned}
$$

In the two-WSRs scenario, in order to enforce the 2nd WSR at NLO one needs the additional constraint $a=M_{V}^{2} / M_{A}^{2}$ (hence restricted to the range $0 \leq a \leq 1$ ). Again, the inequality in the last line flips direction or turns into an equality when, respectively, $M_{A}<M_{V}$ or $M_{V}=M_{A}$.

At LO, $\rho_{T}(t)$ is zero and one has $T_{\mathrm{LO}}=0$. At NLO, where we enforce the $\rho_{S}(t)$ constraints $F_{V} G_{V}=v^{2}$ and $F_{A} \lambda_{1}^{h A}=a v$, we find that $\rho_{T}(t) \stackrel{t \rightarrow \infty}{\longrightarrow} 0$ and obtain the NLO prediction

$$
T=\frac{3}{16 \pi \cos ^{2} \theta_{W}}\left[1+\log \frac{m_{h}^{2}}{M_{V}^{2}}-a^{2}\left(1+\log \frac{m_{h}^{2}}{M_{A}^{2}}\right)\right],
$$

In Fig. 1, we show the compatibility between the experimental determinations for $S$ and $T$ [15] and our NLO determinations in both scenarios. The numerical results in Table 2 show that the precision 
electroweak data requires resonance masses over the $\mathrm{TeV}$ and the $h W W$ coupling to be close to the SM one $\left(a^{\mathrm{SM}}=1\right)$, in agreement with present LHC bounds [22].

To conclude, we emphasize that, remarkably, just by considering the experimental $m_{h}$ (the only LHC input) and the EW precision observables (LEP input), the allowed region concentrates around $a \simeq 1$ for reasonable values of the splitting $M_{V} / M_{A} \sim O(1)$ (see Fig. 1 and Table 2).

\section{References}

[1] S. Weinberg, Physica A96 (1979) 327; J. Gasser and H. Leutwyler, Annals Phys. 158 (1984) 142; Nucl. Phys. B 250 (1985) 465; Nucl. Phys. B 250 (1985) 517.

[2] T. Appelquist and C. W. Bernard, Phys. Rev. D 22 (1980) 200.

[3] A. C. Longhitano, Phys. Rev. D 22 (1980) 1166; Nucl. Phys. B 188 (1981) 118.

[4] Maria J. Herrero and Ester Ruiz Morales, Nucl.Phys. B418 (1994) 431.

[5] R.L. Delgado, A. Dobado, M.J. Herrero, J.J. Sanz-Cillero, JHEP 1407 (2014) 149.

[6] J. Hirn and J. Stern, Phys.Rev. D73 (2006) 056001; G. Buchalla and O. Catà, JHEP 1207 (2012) 101.

[7] D. Espriu and B. Yencho, Phys. Rev D 87 (2013) 055017; D. Espriu, F. Mescia and B. Yencho, Phys. Rev D 88 (2013) 055002; D. Espriu and B. Mescia, Phys.Rev. D90 (2014) 015035; R. L. Delgado, A. Dobado, F. J. Llanes-Estrada, J.Phys. G41 (2014) 025002; JHEP 1402 (2014) 121.

[8] G. Ecker et al., Nucl. Phys. B 321 (1989) 311; G. Ecker et al., Phys. Lett. B 223 (1989) 425.

[9] M. E. Peskin and T. Takeuchi, Phys. Rev. D 46 (1992) 381; Phys. Rev. Lett. 65 (1990) 964.

[10] A. Orgogozo and S. Rychkov, JHEP 1203 (2012) 046.

[11] T. Appelquist and F. Sannino, Phys. Rev. D 59 (1999) 067702.

[12] C. W. Bernard, A. Duncan, J. LoSecco and S. Weinberg, Phys. Rev. D 12 (1975) 792.

[13] S. Weinberg, Phys. Rev. Lett. 18 (1967) 507.

[14] A. Pich, I. Rosell and J.J. Sanz-Cillero, Phys.Rev.Lett. 110 (2013) 181801; JHEP 1401 (2014) 157.

[15] M. Baak et al., Eur. Phys. J. C 72 (2012) 2205; http://gfitter.desy.de/; LEP Electroweak Working Group, http://lepewwg.web.cern.ch/LEPEWWG/.

[16] S. Matsuzaki et al., Phys. Rev. D 75 (2007) 073002; R. Barbieri et al., Phys. Rev. D 78 (2008) 036012; O. Catà and J.F. Kamenik, Phys. Rev. D 83 (2011) 053010; A. Orgogozo and S. Rychkov, JHEP 1306 (2013) 014.

[17] J. M. Cornwall, D. N. Levin and G. Tiktopoulos, Phys.Rev. D 10 (1974) 1145, Erratum-ibid. D 11 (1975) 972; C.E. Vayonakis, Lett.Nuovo Cim. 17 (1976) 383; B.W. Lee, C. Quigg and H.B. Thacker, Phys.Rev. D 16 (1977) 1519; G.J. Gounaris, R. Kogerler and H. Neufeld, Phys.Rev. D 34 (1986) 3257.

[18] M.B. Gavela, K. Kanshin, P.A.N. Machado and S. Saa, [arXiv:1409.1571 [hep-ph]].

[19] M. J. Herrero and E. Ruiz-Morales, Phys.Lett. B296 (1992) 397; J. Bijnens and F. Cornet, Nucl. Phys. B 296 (1988) 557; J. F. Donoghue, B. R. Holstein and Y.C. Lin, Phys.Rev. D 37 (1988) 2423;

J. Bijnens, S. Dawson and G. Valencia, Phys. Rev. D 44 (1991) 3555.

[20] R. Barbieri et al., Nucl. Phys. B 409 (1993) 105.

[21] A. Pich, I. Rosell and J.J. Sanz-Cillero, JHEP 1208 (2012) 106.

[22] ATLAS Collaboration, Report No. ATLAS-CONF-2014-009; CMS Collaboration, Report No. CMS-PAS-HIG-14-009. 